\title{
Computational Comparative Homology Based 3D-structure Modelling of the HSp70 Protein from GWD
}

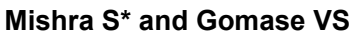

Department of Biotechnology, Mewar University, Chittorgarh, India

\begin{abstract}
Heat shock protein participates in several broad ranges of protein folding processes and control activity of regulatory proteins. Nearly all cells react in a similar manner against the external environmental abrupt changes like heat, chemicals such as analogues of amino acid, ethanol, arsenite, several heavy metals, mitochondrial functional inhibitors, cold, UV light. These all causes several severe, rapid changes in at the level of gene expression. The major significant activity of HSP protein to stabilize newly synthesized protein by securing its right folding, which generally undergone any damaged under cell stress condition. Through overexpression, this protein provides protection to cells against apoptotic cell death. The hsp70 chaperonis targeted as novel anticancer therapeutic candidate for a broad spectrum of tumor types due to its diverse functional role. Homology modeling methodology allowsmodeling the 3Dstructure of the protein using the experimentally determined three dimensional structure of related homologues protein as template. In the current investigation, we have taken the Hsp70 protein and conducted the 3-imensional structure prediction through SwissModel by using the crystal structure as the template. Template search of the desired protein were conducted with Blast and HHBlits against the SMTL and keyed out the highest sequence identity, comparatively, above the other obtained template sequences. The homology modelling was performed and the model protein was evaluated by using the PSVS.
\end{abstract}

Keywords: HSP70; Homology modelling; Protein structure; Molecular modelling

\section{Abbreviations \\ GWD: Guinea Worm Disease; HLA-DR: Human Leukocyte Antigen-Antigen D Related; MHC: Major Histocompatibility Complex; HSp70: Heat Shock Protein 70; BLAST: Basic Local Alignment Search Tool; HHBlits: Lightning-Fast Iterative Protein Sequence Searching by HMM-HMM Alignment; SMTL: SWISS-MODEL Template Library; QMEAN: Qualitative Model Energy Analysis; PSVS: The Protein Structure Validation Software Suite; PDB: Protein Data Bank}

\section{Introduction}

Heat shock protein participates in several broad ranges of protein folding processes and control activity of regulatory proteins. Nearly all cells react in a similar manner against the external environmental abrupt changes like heat, chemicals (like amino acid analogues, ethanol, arsenite, several heavy metals, and certain mitochondrial functional inhibitors, cold [1-2], UV light [3] and during wound healing or tissue remodeling [4] which in turns results inspeedy changes in gene expression levels. The $70-\mathrm{kD}$ class of stress proteins (HSP) draws a major attention because of its diverse range of cellular function. In most of the species it has been found that, this protein is purely stressinducible, the notable exception being primate cells [5]. In human cells, HSP70 is cell cycle regulated [6]. The virtual abundance of the HSPs can be observed in all living organisms starting from bacteria to humans. HSP-70 protein are named according to its molecular weight. Hsp70s $(70-\mathrm{kDa})$ proteins provide assistance in widely distributed protein folding operations, assembling of freshly formed proteins its folding and re-folding action for misfolded protein and aggregated proteins, and controlling the regulatory proteins activity [7-13]. This protein has also performed functions of housekeeping in the cell. In an ATP controlled fashion this protein interacts with hydrophobic peptide segments. A peptide binding domain (PBD) and the amino-terminal ATPase domain $(\mathrm{ABD})$ is the two distinct functional regions of this protein. For the neutral and hydrophobic amino acid residues affinity the PBD region of HSP70 holds a groove, whereas, the C-terminal or
$\mathrm{ABD}$ is rich in alpha helical structure which behaves as a 'lid' for the substrate binding domain. When ATP is bounded with protein the lid remains open and allows peptides to bind and release rapidly but when ADP is bound, the lid remains closed, and peptides are strongly bound to the substrate binding domain. In malignant melanoma this protein is over-expressed in cell [14] but under expression is also seen in renal cell cancer [15]. The extracellular hsp perform a potent route for sending the danger signals to the host immune system in order to respond to an infection. The peptide complexes of hsp protein are also involve in the restricted antigen presentation of MHC classes (I\&II) and enable the enhanced activation of $\mathrm{T}$ cells. The specific interaction of the mammalian cytosolic Hsp70 molecules with HLA-DR molecules, signifies the possibility of transferring the of bond antigenic peptides in the ternary complex into the binding groove of HLA-DR molecules by Hsp70 molecules. Rohrer et al., study suggest that the interaction of Hsp70 and HLA-DR takes place outside the peptide binding groove and is assigned to ATPase domain of heat shock protein 70 molecule, which enable the enhanced presentation of peptide to the antigenic presenting cells and improved $\mathrm{T}$ cells proliferations [16]. The computational method approach is the one of the reliable methodology to generate amino acid sequence into 3-D structure models [17] and a wide range of the approaches are routinely applied for such prediction for many biological applications. Homology modeling is based on the sensible assumption of two homologous proteins shares very standardized alike structures. The name homology modelling conveys exactly what this procedure is about; modeling a structure using homologous model as template (which is usually an exact X-ray or NMR-determined

*Corresponding author: Mishra S, Department of Biotechnology, Mewar University, Chittorgarh, India, Tel: 9560808369; E-mail: sonumishra1014@gmail.com

Received April 29, 2016; Accepted June 16, 2016; Published June 23, 2016

Citation: Mishra S, Gomase VS (2016) Computational Comparative Homology Based 3D-structure Modelling of the HSp70 Protein from GWD. J Health Med Informat 7: 233. doi:10.4172/2157-7420.1000233

Copyright: @ 2016 Mishra S, et al. This is an open-access article distributed under the terms of the Creative Commons Attribution License, which permits unrestricted use, distribution, and reproduction in any medium, provided the original author and source are credited. 
Citation: Mishra S, Gomase VS (2016) Computational Comparative Homology Based 3D-structure Modelling of the HSp70 Protein from GWD. J Health Med Informat 7: 233. doi:10.4172/2157-7420.1000233

Page 2 of 6

structure).In homology modeling it is important that modeler finds a template structure with the highest possible sequence-identity. If the identity between the input sequence and the template structure falls below $40 \%$, the output model is likely to be implausible. Earlier study reveals that about the conservation of the protein structures than protein sequences in the midst of homologues [18].

\section{Methodology}

\section{Retrieval of the protein sequence}

For the present study heat shock protein 70 [Dracunculus medinensis] the protein sequence was retrieved from the NCBI having 647 amino acids sequence [ADI54942.1] (Table1) [19].

\section{Template search and selection}

For the target protein, the templates were search and identified by performing BLAST and HHBlits against the SWISS-MODEL template library (SMTL) [20-21]. A total of 96 templates were opted .For each identified template, the quality of the template's was predicted from features of the target-template alignment.Among of them; $3 \mathrm{c} 7 \mathrm{n} .1$ chain B with highest sequence identity (Sequence identity: 88.45), and lowest two sequence similarity found are $2 \mathrm{v} 7 \mathrm{z} .2$ chain A and $2 \mathrm{v} 7 \mathrm{z} .1$ chain A with sequence identity: 88.40 value had then selected as template for building model.

\section{Molecular modeling of protein}

The methodological strategy used in the model building was based on the target-template alignment using Promote-II. The template were selected based on the maximum sequence similarity. The $3 \mathrm{c} 7 \mathrm{n} .1$ found to have the maximum sequence similarity and taken as template and alignment were performed between the both the targets and template. The coordinates which are found as conserved between the targets and templates are copied from the template to the model. Using a fragment library, insertion and deletion are remodelled and the rebuilding of the side chains was performed. Finally, the geometry of the resulting model is regularized by using a force field. The obtained model is visualized with Deep Viewer. The global and per-residue model quality has been assessed using the QMEAN4 scoring function [22].

\section{Ligand modelling}

The number of the ligands which were found to be present in the template structure (Ligands such as SO4, BEF-ADP, ADP) are reassigned by homology in order of modelling, where the ligands shouldn't be clashing with the target protein and the residue which are in contact with the ligand were conserved among the target and the template.

\section{The protein model statistical assessment}

The protein model statistical Assessment were performed via the protein structure validation software suite (PSVS). PSVS were used for those protein structures which are generated from NMR, X-ray crystallographic and homology modelling methods. PSVS incorporates analyses from numerous widely-used structure quality evaluation tools, including RPF, PROCHECK, MolProbity, Verified 3D, Prosa II, the PDB validation software and various structure validation tools [23]. PSVS provides a standard constraint analyses, statistics on the PDB validation goodness-of-fit between structures and experimental data, Z-score values and knowledge- based structure quality scores in a standardized format suitable for database integration.

\section{Visualization of 3D model}

The generated model was visualized in $3 \mathrm{D}$ using the RasMOl molecular 3D viewer. The RasMol generated model information regarding chain, atoms, groups and bonds of 3D model were extracted and analysed [24].

\section{Results and Interpretation}

For the recognized 3-D protein structure model, the complete assessment were performed, analyzed and described into the following leads

Template validation: For each indentified template, the template's quality has been predicted from the features of the target-template alignment as shown in Table 2. The templates with the highest quality have been selected for the model building. Template $3 \mathrm{c} 7 \mathrm{n} .1 . \mathrm{B}$ validations for the generated model of Hsp70 protein was analyzed and were employed structure analysis for template chain. The multiple sequence alignment result of target protein sequence and the template $3 \mathrm{c} 7 \mathrm{n} .1$ chain B is found to be $88.45 \%$ identity by $\mathrm{HHb}$ lists as shown in the Figure 1.

Model analysis: HSP70 protein model structure was prepared for the target-template alignment, because the template $3 \mathrm{c} 7 \mathrm{n} .1$ has the highest quality and alignment with the target (Table 3), therefore selected for modelling as shown in Figure 2.

The result of PSVS provides the stereo-chemical property of the model. The molecular weight of the model is 131894. RMS deviation of the bond angle is $1.4^{\circ}$. Number of close contacts (within $2.2 \AA$ ): 0 and the bond lengths is $0.011 \AA$. With respect to mean and standard deviation for a set of $252 \mathrm{X}$-ray structures $<500$ residues, of resolution $\leq 1.80 \AA$, $\mathrm{R}$-factor $\leq 0.25$ and $\mathrm{R}$-free $\leq 0.28$; a positive value indicates

MAKHNAVGIDLGTTYSCVGVFMHGKVEIIANDQGNRTTPSYVAFTDTERLIGDAAKNQVAMNPNNTV FDAKRLIGRRFDDPAVQADMKHWPFKVINAEGSKPKVQVEYKGETKTFTPEEISSMVLLKMKETAEAF LGSTVKDAVVTVPAYFNDSQRQATKDAGAIAGLNVLRIINEPTAAAIAYGLDKKGHGERNVLIFDLGG GTFDVSILTIEDGIFEVKSTAGDTHLGGEDFDNRMVNHFVAEFKRKHKKDLSTNPRALRRLRTACERAK RTLSSSSQASIEIDSLFEGIDFYTNITRARFEELCADLFRSTMDPVEKALRDAKMDKSQMHDIVLVGGST RIPKVQKLLSDFFSGKELNKSINPDEAVAYGAAVQAAILSGDKSEAVQDLLLLDVAPLSLGIETAGGVM TALIKRNTTIPTKTAQTFTTYSDNQPGVLIQVFEGERAMTKDNNLLGKFELSGIPPAPRGVPQIEVTFDID ANGILNVSAQDKSTGKQNKITITNDKGRLSKDEIERMVQEAEKYKADDEAQKDRIAAKNALESYAFN MKQTIDDEKLKDKLSADDRKKIEDKCDEIIKWLDRNQTAEKDEFEHHQKELEAVCNPIITKMYQSAGG MPGNPGGFPGGGAPGGGHQGGGGPTIEEVD

Table 1: Primary amino acid sequences of target protein for which template were searched for models building. 
Citation: Mishra S, Gomase VS (2016) Computational Comparative Homology Based 3D-structure Modelling of the HSp70 Protein from GWD. J Health Med Informat 7: 233. doi:10.4172/2157-7420.1000233

Page 3 of 6

a 'better' score Selected residues: 3A-547B. The model protein 3D structure is shown in the Figure 3.

Ramachandran plot statistics: The Ramachandran plot displays the psi and phi backbone conformational angles for the each residue in the target protein Hsp70 as shown in the Figure 4. The displayed darkest region as 'red' in colour and correspond to the "core" region and represent the most favourable combination of phi-psi values. Few residues found in allowed region. The percentage of residue in the core regions are described as follows in Table 4.

PROCHECK output: The protein sterochemical quality was analysed. The PDB structure of HSp70 protein was examined by PROCHECK tool. Procheck G-factor evaluated (Figure 5) probability of dihedral angles of a residue types to be within a given range as below:

(a) Procheck G factor for phi-psi for ordered residue overall is -0.659 .

(b) Procheck g-factor for all dihedral angles for ordered residue overall is -0.619 .

\section{Output from MolProbity}

The MolProbity server (in PSVS server) is a one of the worthful structure validation tool in the final stage of structure refinement.VDW violations from MAGE calculate MAGE VdW clashscore: Mean 38.49; SD: 0.0000 . MolProbity clash score and visualize atomic overlap and beta position deviations (Figure 6).

\section{PDB validation software output}

After the PDB validation software analysis 3.5 Angstroms are consider for hydrogen bonding in the asymmetric unit and 2.2 Angstroms are considered as close contact for heavy atoms in same asymmetric unit. Distances smaller than 2.2 Angstroms are considered as close contacts. The RMS deviation for covalent bonds relative to the standard dictionary is 0.011 Angstroms (Tables 5 and 6).

The following table contains a list of the covalent bondsgreater than $6.0^{*} \mathrm{RMSD}$.

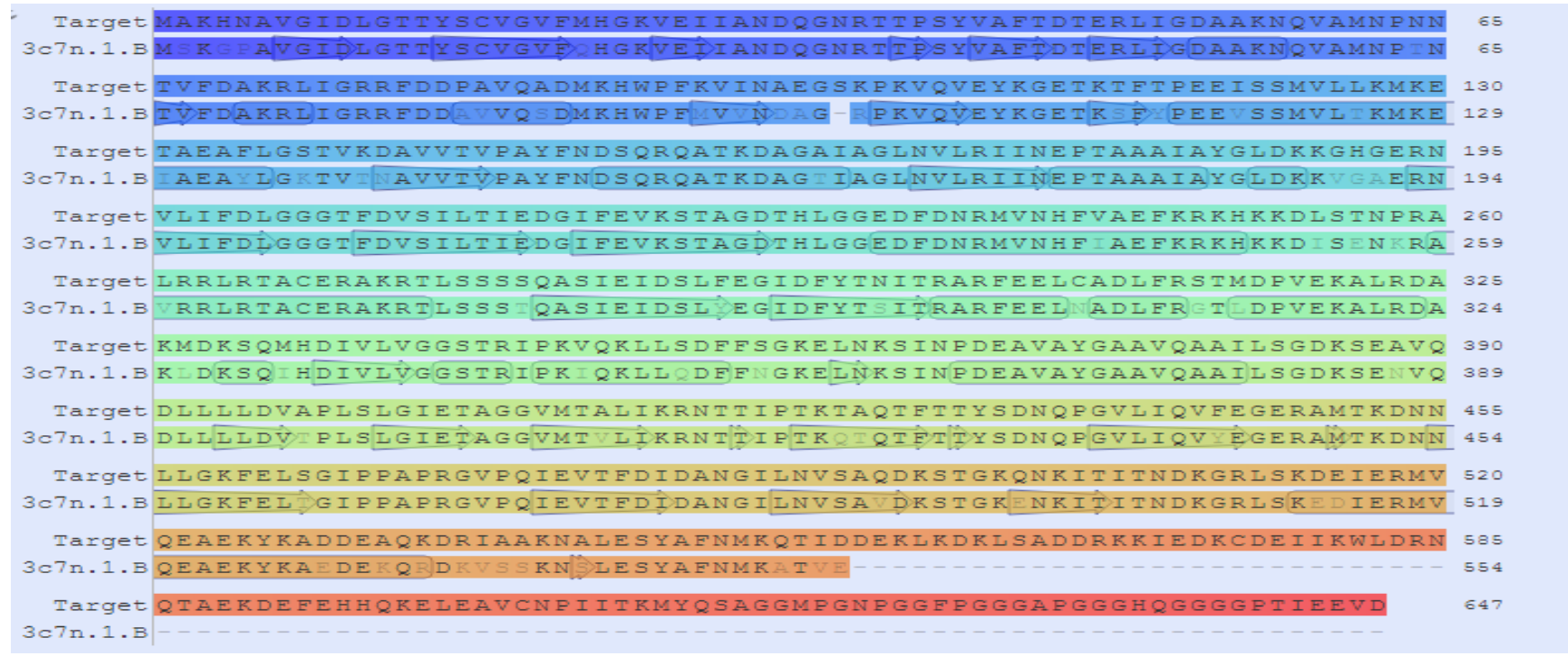

Figure 1: Hsp70 protein sequence and template 3c7n.1 chain B multiple sequence alignment result with $89.06 \%$ of identity obtained through HHblits.
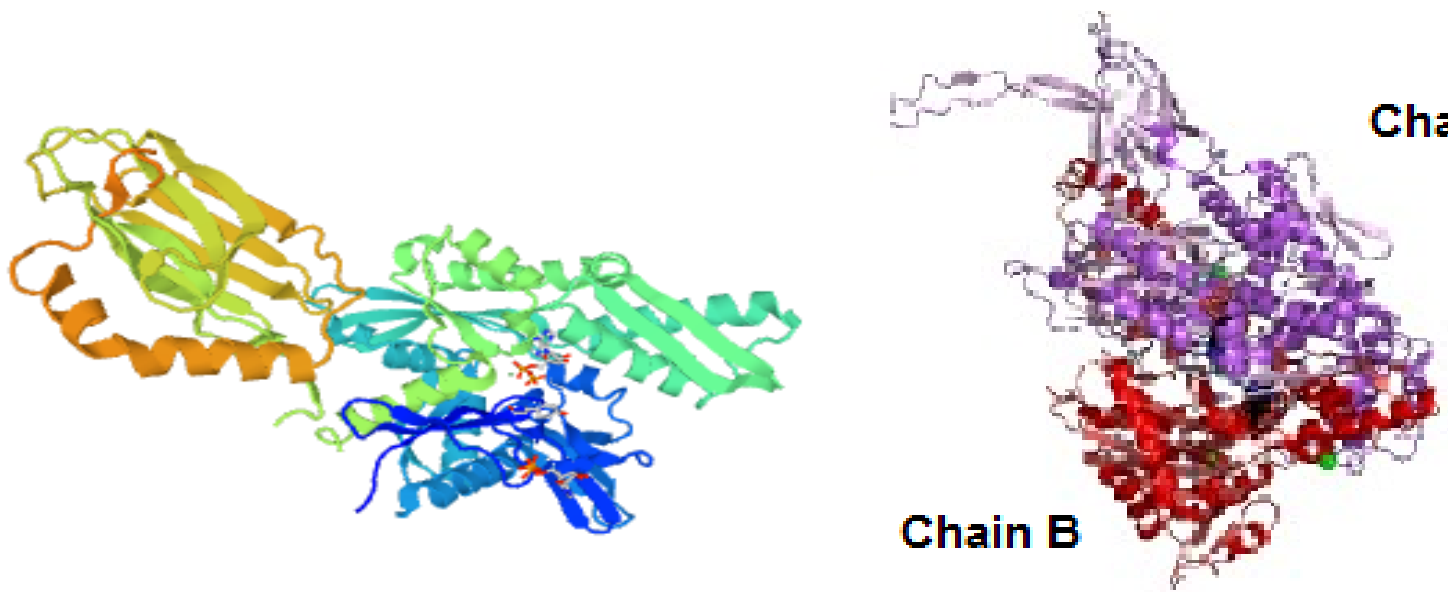

Figure 2: The template 3c7n.1 chain B PDB structure in ribbon form (Chain B-coloured as 'Red' and chain A as 'Purple'). 
Citation: Mishra S, Gomase VS (2016) Computational Comparative Homology Based 3D-structure Modelling of the HSp70 Protein from GWD. J Health Med Informat 7: 233. doi:10.4172/2157-7420.1000233

Page 4 of 6

\begin{tabular}{|l|l|l|l|l|l|l|l|l|l|}
\hline Template & Seq Identity & Oligo-State & Found by & Method & Resolution & Sequence similarity & Range & Coverage & \\
\hline 3 c7n.1.B & 88.45 & Hetero-oligomer & HHblits & X-ray & $3.12 \AA$ & 0.57 & $1-555$ & 0.86 \\
\hline
\end{tabular}

Table 2: 3c7n.1 chain Bwith highest sequence similarity choosen as the template for the protein modeling.

\begin{tabular}{|l|l|}
\hline Model Properties & Data \\
\hline Number of Atoms & 4403 \\
\hline Number of Bonds & 4343 \\
\hline
\end{tabular}

Table 3: The template model important information.

\begin{tabular}{|c|c|c|}
\hline & From PROCHECK & From Richardson Lab's Molprobity \% target \\
\hline Most favoured regions & $81.40 \%$ & $85.8 \%$ \\
\hline Additional allowed regions & $16.60 \%$ & $10.9 \%$ \\
\hline Generously allowed region & $1.50 \%$ & N/A \\
\hline Disallowed regions & $0.50 \%$ & $3.3 \%$ \\
\hline Structure quality-factors-overall statistics & Mean Score & SD \\
\hline Procheck G-factor (phi/psi only) & -0.66 & N/A \\
\hline Procheck G-factor (phi/psi only) & -2.28 & N/A \\
\hline MollProbityClashscore & -0.62 & -3.67 \\
\hline
\end{tabular}

Table 4: The percentage of residue in the core regions.

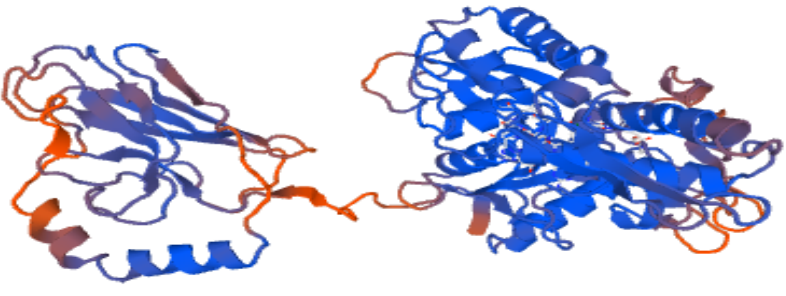

Figure 3: The model of target protein with template.

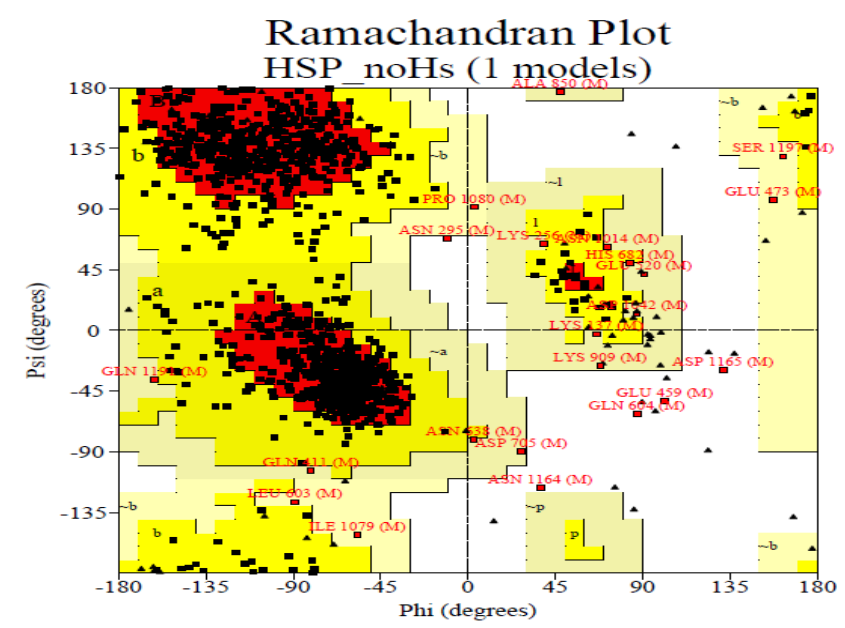

Figure 4: Showing the Ramachandran plot of modelled protein Hsp70.
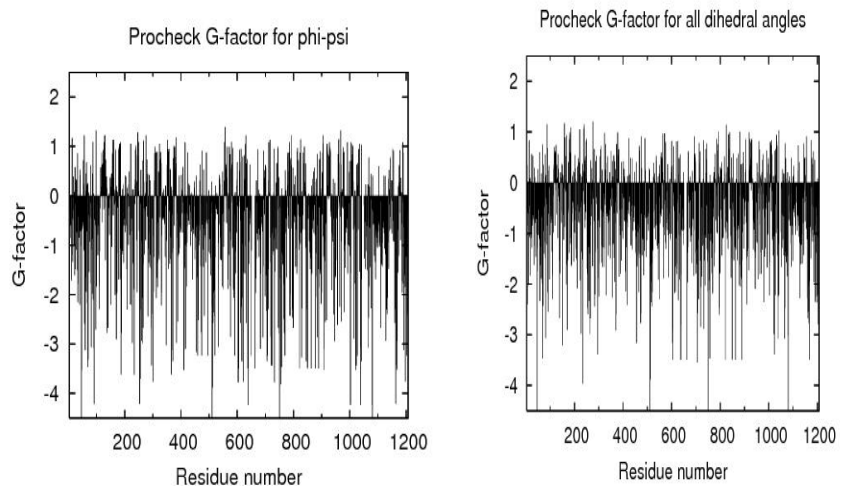

Figure 5: Procheck G-factor evaluated probability of dihedral angels of a residue.

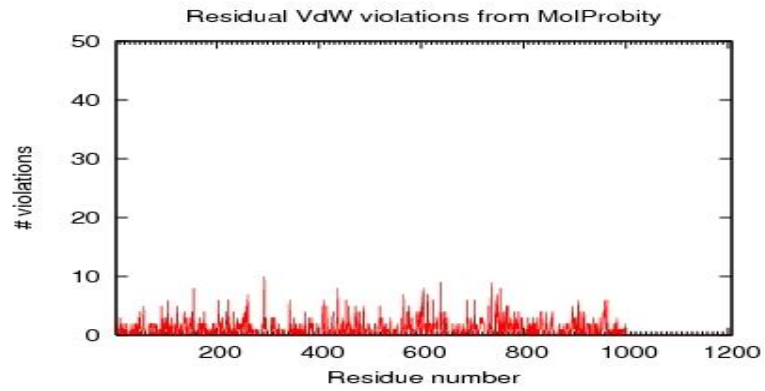

Figure 6: Showing VDW violations from MAGE calculate MolProbity clash score. 
Citation: Mishra S, Gomase VS (2016) Computational Comparative Homology Based 3D-structure Modelling of the HSp70 Protein from GWD. J Health Med Informat 7: 233. doi:10.4172/2157-7420.1000233

Page 5 of 6

\begin{tabular}{|c|c|c|c|c|c|}
\hline Deviation & Residue Name & Chain ID & Sequence Number & AT1 - AT2 Distance & Bond Value \\
\hline 0.094 & LYS & A & 197 & CD - CE & 1.614 \\
\hline 0.068 & PRO & A & 512 & CB - CG & 1.52 \\
\hline 0.141 & ARG & B & 301 & CG - CD & 1.492 \\
\hline
\end{tabular}

Table 5: List of the covalent bonds greater than 6.0*RMSD. The RMS deviation for covalent angles relative to the standard dictionary is 1.4 degrees.

\begin{tabular}{|c|c|c|c|c|c|}
\hline Deviation & Residue Name & Chain ID & Sequence Number & AT1 - AT2 - AT3 Distance & Bond Value \\
\hline 8.4 & THR & A & 189 & N - CA - C \\
\hline-9.8 & LYS & A & 219 & N - CA - C & 119.6 \\
\hline-9.1 & LYS & A & 431 & N - CA - C & 111.2 \\
\hline 9.9 & GLY & B & 224 & N - CA - C \\
\hline
\end{tabular}

Table 6: List of the covalent bond angles greater than $6.0^{*} \mathrm{RMSD}$.

\section{Chirality}

The chirality checking was performed and it was found that, that there are no incorrect carbon chiral centers.

\section{Conclusion}

In this current study, we have modelled a 3D structure of the hsp70 protein by homology modeling and visualized with the help of online computational tools. 3-D models of hsp70 protein shows significant amino acid sequence similarity with the target sequence. Homology modelling suggested the similarity between targets: Template sequences. In this model, a template is a homologous protein that can be identified by a sequence similarity with target, and $89.45 \%$ identity was identified. Protein validation prediction indicates about the region where residues are present. Ramachandran Plot analysis from PROCHECK which indicated the maximum of the residues present in most favoured region, i.e., $81.40 \%$ of the residues were found in the most favoured region and form Richardson's lab Molprobity present $85.8 \%$ favoured regions for the selected residues. 3D structure of nematode protein-hsp70 protein structure reported extremely in any cases in GWD. In the present current study we used GWD-HSP70 protein sequence as target with $3 \mathrm{c} 7 \mathrm{n} .1$ template for the modeling of the target protein sequence, because at the point of the template selection procedure, we found that the target protein template provides more sequence identity in comparison to other founded template sequences. This study may provide the future prospects to illustrate consideration towards a computational approach for 3D molecular modelling and computer generated model are expected to be the most accurate model but it can't be a substitute of a crystal structure. The HSP70 3-D model representation will prove to be a useful model for exploiting in the dracunculiasis disease outbreak database and residue or derivatives relationship and experimental verification in future and could be helpful in drug designing and development.

\section{References}

1. Matz JM, Blake MJ, Tatelman HM, Lavoi KP, Holbrook NJ (1995) Characterization and regulation of cold-induced heat shock protein expression in mouse brown adipose tissue. Am J Physiol 269: R38-R47.

2. Wong HR (1999) Heat shock proteins. Facts, thoughts, and dreams. A. De Maio. Shock 11:1-12, 1999. Shock 12: 323-325.

3. Cao Y, Ohwatari N, Matsumoto T, Kosaka M, Ohtsuru A, et al. (1999) TGFbeta1 mediates $70-\mathrm{kDa}$ heat shock protein induction due to ultraviolet irradiation in human skin fibroblasts. Pflugers Arch 438: 239-244.
4. Laplante AF, Moulin V, Auger FA, Landry J, Li H, et al. (1998) Expression of heat shock proteins in mouse skin during wound healing. $J$ Histochem Cytochem 46: 1291-1301.

5. Welch WJ, Suhan JP (1986) Cellular and biochemical events in mammalian cells during and after recovery from physiological stress. J Cell Biol 103: 2035-2052.

6. Milarski KL, Morimoto RI (1986) Expression of human HSP70 during the synthetic phase of the cell cycle. Proc Natl Acad Sci U S A 83: 9517-9521.

7. Bukau B, Deuerling E, Pfund C, Craig EA (2000) Getting newly synthesized proteins into shape. Cell 101: 119-122.

8. Hart FU, Hart HM (2002) Molecular chaperones in the cytosol: from nascent chain to folded protein. Science 295: 1852-1858.

9. Young JC, Barral JM, Ulrich Hartl F (2003) More than folding: localized functions of cytosolic chaperones. Trends Biochem Sci 28: 541-547.

10. Neupert W, Brunner M (2002) The protein import motor of mitochondria. Nat Rev Mol Cell Biol 3: 555-565.

11. Ryan MT, Pfanner N (2001) Hsp70 proteins in protein translocation. Adv Protein Chem 59: 223-242.

12. Pratt WB, Toft DO (2003) Regulation of signaling protein function and trafficking by the hsp90/hsp70-based chaperone machinery. Exp Biol Med (Maywood) 228: 111-133.

13. Toft DO (1999) Control of hormone receptor function by molecular chaperones and folding catalysts. In: Molecular Chaperones and Folding Catalysts. Regulation, Cellular Function and Mechanism. Bukau B, Harwood Academic Publishers, Amsterdam, pp: 313-327.

14. Ricaniadis N, Kataki A, Agnantis N, Androulakis G, Karakousis CP (2001) Long-term prognostic significance of HSP-70, c-myc and HLA-DR expression in patients with malignant melanoma. Eur J Surg Oncol 27: 88-93.

15. Ramp U, Mahotka C, Heikaus S, Shibata T, Grimm MO, et al. (2007) Expression of heat shock protein 70 in renal cell carcinoma and its relation to tumor progression and prognosis. Histol Histopathol 22: 1099-1107.

16. Rohrer KM, Haug M, Schwörer D, Kalbacher H, Holzer U (2014) Mutations in the substrate binding site of human heat-shock protein 70 indicate specific interaction with HLA-DR outside the peptide binding groove. Immunology 142 237-247.

17. Bagchi P (2014) "Ayurinformatics : Selecting the best ligand for schizophrenic ProteinD (3) Dopamine Receptor (DRD3) from the Compund of Valerianawallichi Asparagus racemosus and Acoruscalamus". Int J Pharm Bio Sci 1: 1-10.

18. Chothia C, Lesk AM (1986) The relation between the divergence of sequence and structure in proteins. EMBO J 5: 823-826.

19. http://www.ncbi.nlm.nih.gov/ 
Citation: Mishra S, Gomase VS (2016) Computational Comparative Homology Based 3D-structure Modelling of the HSp70 Protein from GWD. J Health Med Informat 7: 233. doi:10.4172/2157-7420.1000233

Page 6 of 6

20. http://swissmodel.expasy.org/

21. Altschul SF, Madden TL, Schäffer AA, Zhang J, Zhang Z, et al. (1997) "Gapped BLAST and PSI-BLAST: a new generation of protein database search programs." Nucleic Acids Res 25: 3389-3402.

22. Benkert P, Biasini M, Schwede T (2011) Toward the estimation of the absolute quality of individual protein structure models. Bioinformatics 27: 343-350.

23. http://psvs-1_5-dev.nesg.org/results/HSP/OUTPUT.html

24. http://rasmol.org/ 